DOI: https://doi.org/10.14311/TPFM.2022.007

\title{
AN INLET FLOW ANGLE EFFECT ON A HORSE-SHOE VORTEX FORMA- TION
}

\author{
E. Flídr ${ }^{1}$, T. Jelínek ${ }^{1}$, Z. Ilich ${ }^{1}$, M. Němec ${ }^{1}$ \\ ${ }^{1}$ VZLÚ - Czech Aerospace Research Centre, Beranových 130, 19905 Prague - Letňany, Czech \\ Republic
}

\begin{abstract}
The static pressure distribution on the end-wall of a linear blade cascade for different inlet flow angles is investigated in this contribution. The experiments were performed for constant values of Reynolds number $\left(\operatorname{Re}_{2, \text { is }}=4.5 \times 10^{5}\right)$ and Mach number $\left(M_{2, \text { is }}=0.4\right)$. These data are presented in the form of static pressure gradients. The obtained quantitative results are supported by surface flow visualization.
\end{abstract}

Keywords: secondary flow, linear blade cascade, static pressure measurement, surface flow visualization.

\section{Introduction}

The secondary flow is a complicated phenomenon, where three-dimensional flow structures occur. This kind of flow has been investigated by many authors from different perspectives. Theoretical research was conducted by Hawthorne [1], Marris [2, 3, 4], Horlock \& Lakshminarayana [5], or by Came \& Marsh [6]. Note that these investigations were not able to describe secondary flow when the boundary layer separation occurred. Hawthorne [1] derived the relationship for the vorticity in the stream-wise direction in the form:

$$
\frac{\partial}{\partial s}\left(\frac{\omega_{s}}{q}\right)=\frac{2 \omega_{n}}{q \mathcal{R}}
$$

where $\omega_{s}$ and $\omega_{n}$ are vorticities in the stream-wise direction, and in the normal direction, respectively. $q$ is the velocity scalar and $\mathcal{R}$ is the streamline curvature. This relationship was derived based on flow kinematics. The equation analysis shows that the secondary vorticity can not be generated without the streamlined curvature and the normal vorticity. Marris [2] performed a similar derivation and obtained equation where the additional term $-\frac{l}{q^{2}} \operatorname{rot}(\mathbf{u} \times \Omega)$ appears. This term allows the stream-wise vorticity generation without the streamline curvature. However, even this modified equation cannot predict the effect of the Reynolds number on the secondary flow because the viscous term is not present. By applying curl on the Navier-Stokes equation for incompressible flow in intrinsic coordinates, one can obtain:

$$
\frac{\mathrm{D} \omega_{s}}{\mathrm{D} \tau}=\omega_{s} \frac{\partial u}{\partial s}+\omega_{n} \frac{\partial u}{\partial n}+\omega_{z} \frac{\partial u}{\partial z}+\nu \nabla^{2} \omega_{s},
$$

Oncoming flow
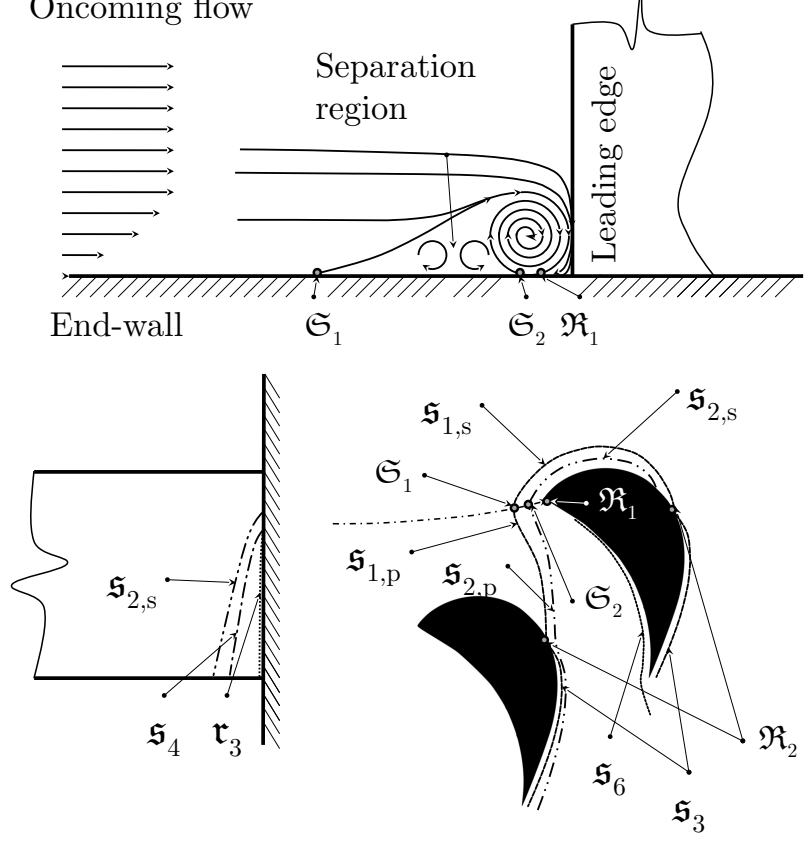

Figure 1: Formation of secondary flow. where the effect of viscosity on the generated stream-wise vorticity is obvious. The effect of the inlet flow angle can be deduced from this equation as well. Only $\omega_{n}$ is present at the cascade inlet 
due to the boundary layers on the end-wall. Therefore stream-wise vorticity will be dependent on the inlet boundary layer as well as on the velocity gradients $\frac{\partial u}{\partial n}$. An increase of the inlet flow angle results in the larger velocity gradient $\frac{\partial u}{\partial n}$ and therefore in the enhancement of stream-wise vorticity.

These theoretical results were investigated experimentally as well. Hodson \& Dominy $[7,8]$ investigated the secondary flow in the linear blade cascade at the design as well as at the off-design conditions. They focused on the effects of both Reynolds number and inlet flow angle. Similar studies were performed by Perdichizzi and Dossena [9], and Perdichizzi [10], where, in addition, the effect of compressibility was studied. It can be concluded that the secondary vorticity was enhanced when the studied parameter was enhanced as well. Note that the facilities where these studies were performed are not able to vary both Mach number and Reynolds number independently. Moreover, most of the papers focus on the measurement at the cascade outlet. The formation of the secondary flow within the blade cascade starts with the separation of the inlet boundary layer in front of the cascade, as is shown in Fig 1. The horseshoe vortex has two legs. The pressure leg is driven into the blade channel due to the pressure gradient, meanwhile, the suction leg is attached to the blade suction surface for the same reason.

\section{Aim of the work}

In this contribution, the secondary flow is investigated based on the static pressure measurement at the linear blade cascade end-wall. The effect of flow turning on the secondary flow in the cascade is studied. The surface flow visualization was performed as well to support the measured data.

\section{Experimental apparatus, setup and methods}

\subsection{Apparatus and setup}

The experiments were performed in the low-pressure circular wind tunnel in the VZLU laboratory of high-speed aerodynamics at Palmovka. The flow in the tunnel is driven by the twelve-stage radial compressor. This type of tunnel allows independent variation of both Reynolds and Mach numbers. The Mach number is set by the rotational speed of the compressor, while the Reynolds number is changed by the pressure in the tunnel. The pressure is varied by a set of vacuum pumps.

The inlet flow angle (flow turning) can be set by a pair of semi-shaped nozzles positioned in front of the cascade. The cascade itself is composed of individual prismatic blades assembled in the test section between two acrylic windows. The exception was the two blades used for the static pressure measurement. These two blades were printed by the PLA method as one piece, together with the part of the end wall and with the static pressure taps, see Fig. 2.

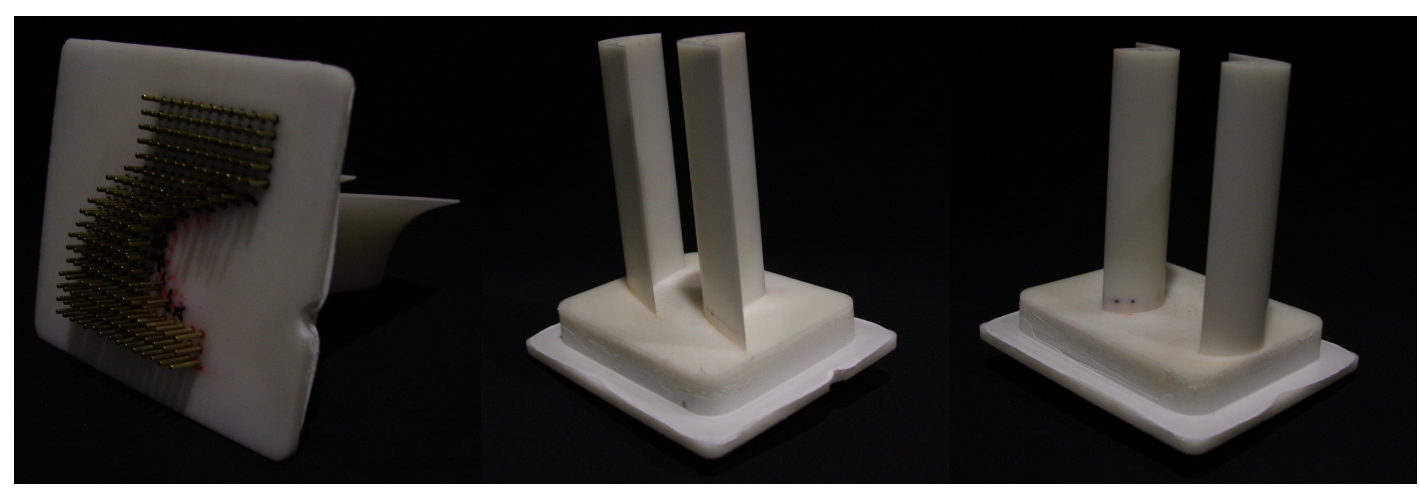

Figure 2: 3D printed cascade section with the static pressure taps.

The presented experiments were conducted at the constant values of Mach number $\left(M_{2, \text { is }}=0.4\right)$ and Reynolds number $\left(R e_{2 \text {,is }}=4.5 \times 10^{5}\right)$. These values were set with respect to the tested blades (nozzle blades of a high-pressure part of a steam turbine) and with respect to the operational 
conditions of the tunnel. The cascade was assembled into the pitch to chord ratio $t / c=1.00$. The flow turning effect was investigated for different inlet flow angles $\alpha_{1} \in(20,55)^{\circ}$. This study is part of the more complex research where even the effects of Reynolds number and Mach number are investigated.

\subsection{Methods}

The phenomenon under investigation was studied based on static pressure measurements at the end-wall of the wind tunnel. One of the blade channels was equipped with 192 static pressure taps in several rows and columns with a distance of $5 \mathrm{~mm}$ in each direction $(x$ and $y)$. Their position was chosen with respect to the studied phenomenon and will be obvious from the graphs in section 4. The results are presented in terms of dimensionless static pressure coefficients defined:

$$
C_{\mathrm{ps} i}=\frac{p_{01}-p_{\mathrm{s} i}}{Q}
$$

where $Q$ is the dynamic pressure, $p_{\mathrm{s}_{i}}$ are the static pressures measured on the end-wall and $p_{01}$ is the stagnation pressure measured in the relaxation chamber at the cascade inlet. The static pressure gradients normalized by the dynamic pressure calculated as:

$$
\frac{1}{Q} \frac{\partial p_{\mathrm{s}}}{\partial x_{j}}=\frac{1}{Q}\left(\frac{\partial p_{\mathrm{s}}}{\partial x} ; \frac{\partial p_{\mathrm{s}}}{\partial y}\right)
$$

where $x$ and $y$ are the coordinates defined in the circumferential $(x)$ and axial direction $(y)$, were evaluated as well.

The flow visualization was performed according to the experiments performed in the past and published by Flídr et al. in [11]. In short, solutions of two powder dyes were used to visualize the flow phenomena on the blade suction surface as well as on the cascade end-wall. The dyes were injected from the taps positioned $3 \mathrm{~cm}$ in front of the cascade and from the taps placed behind the leading edge on the blade suction surface.

\subsection{Measurement technique and uncertainty}

The flow regimes were set according to the measurement of state variables i.e. pressure (static and stagnation), the temperature at the cascade inlet and outlet. The barometric pressure was measured by digital pressure transducer Druck DPI 145 with the uncertainty of $0.013 \%$ FS. The individual pressures were measured by the differential pressure transducers with the uncertainty of $0.1 \%$ RDG. Temperature and relative humidity were both measured by the hygrometer Sensorica Humistar HTP-1 with the precision of $R H \pm 2 \%$ and with the precision of $T \pm 0.3 \mathrm{~K}$. The static pressures on the end-wall were measured by pressure scanners Scanivalve ZOC33/64xX2 with uncertainty $0.08 \%$ FS. Mach number was set with uncertainty under $1 \%$ and Reynolds number with uncertainty under $2 \%$. The evaluation of the uncertainty was performed for the confidence level of $95 \%$ (standard deviation $\pm 2 \sigma$ ).

\section{Results and discussion}

\subsection{Static pressure measurement}

The static pressure gradients are plotted in Fig. 3. The contours in the graphs represent the distribution of the static pressure coefficient $C_{\mathrm{ps} i}$.

The distributions of the pressure gradients show the effect of the inlet flow angle on the horseshoe vortex pressure leg. This vortex entered into the area under investigation on the position approximately $x, y \approx(-10,55)$ and is driven by the pressure gradient between both blade surfaces (pressure and suction). The vortex consumed the low momentum fluid from the end-wall boundary layer and was, moreover, fed by the crossflow in the passage. This mechanism was responsible for the formation of the so-called passage vortex. The reattachment of the passage vortex occurs at the point $x, y \approx(-32,20)$ in all studied cases. These findings are in disagreement with the results published earlier by Flídr et al. in [12], where 


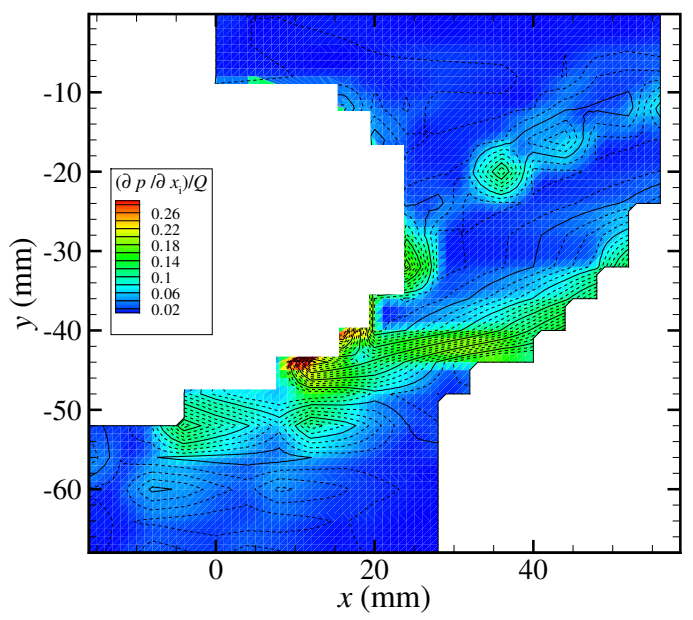

(a) $\alpha_{1}=20^{\circ}$

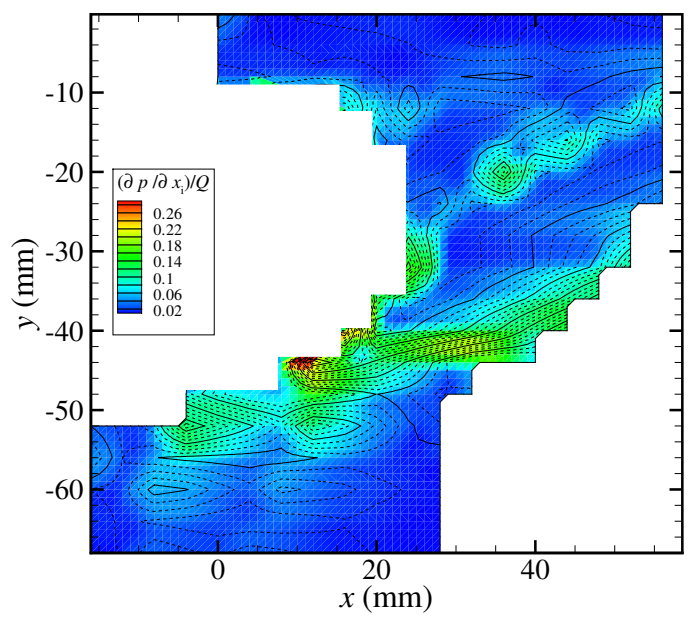

(c) $\alpha_{1}=40^{\circ}$

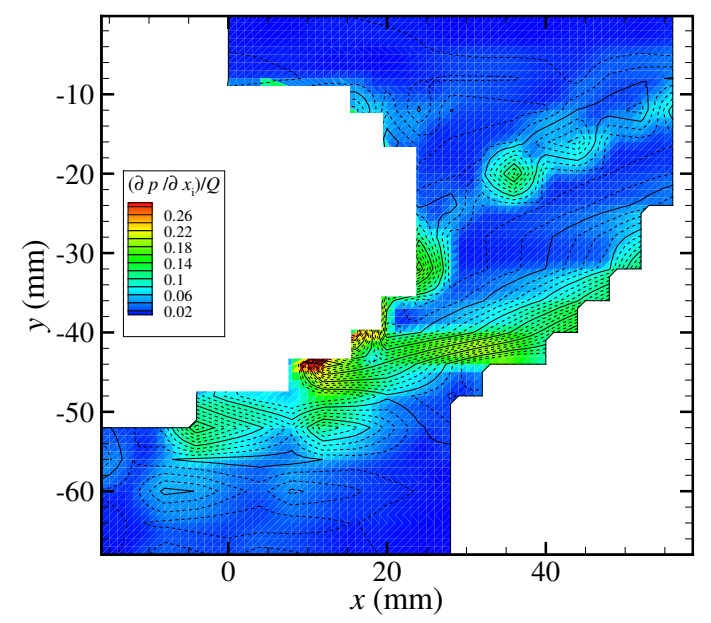

(b) $\alpha_{1}=30^{\circ}$

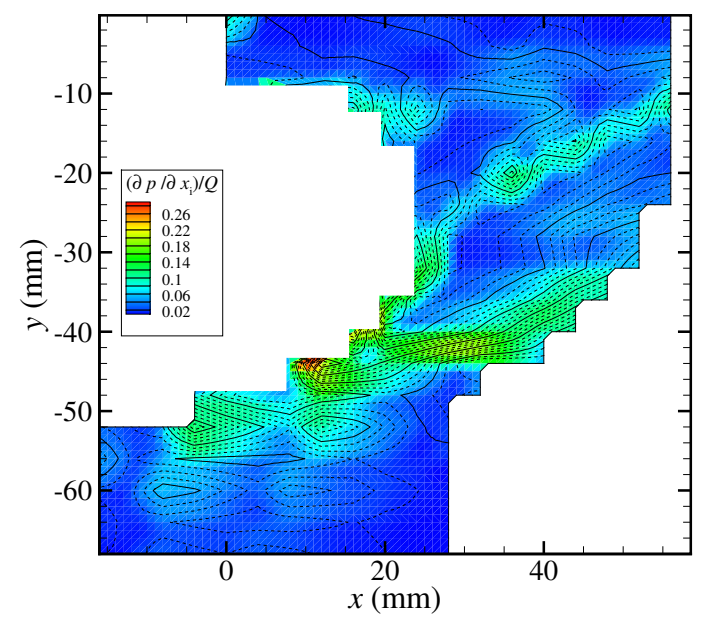

(d) $\alpha_{1}=50^{\circ}$

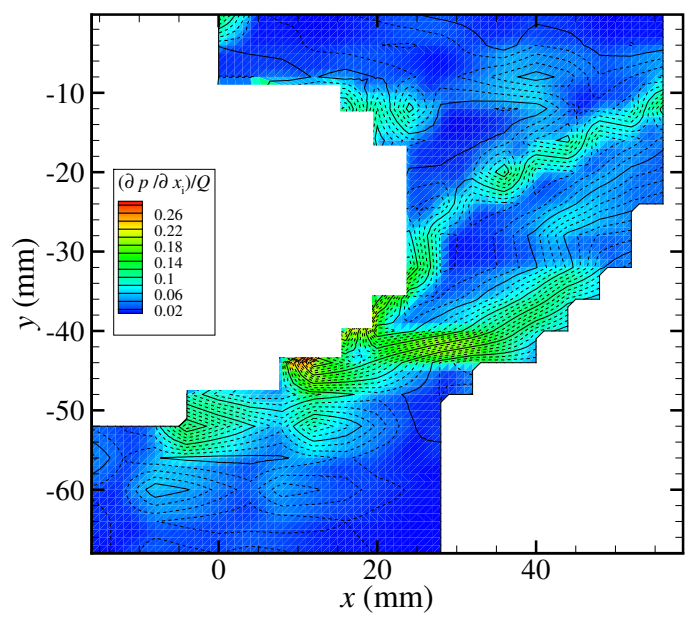

(e) $\alpha_{1}=55^{\circ}$

Figure 3: The static pressure gradient evolution for $M_{2, \text { is }}=0.40$ and $R e_{2, \text { is }}=4.5 \times 10^{5}$. 
the increase of inlet flow angle resulted in a movement of the reattachment point $\mathfrak{R}_{2}$ toward the leading edge of the blade. The reason for this disagreement can be found in a different type of cascade (guide vanes were investigated in the past), where the impact of inlet flow angle on secondary flow was considerably higher compared to this case. Another reason is that a different pitch to chord ratio was studied in the [12] $(t / c=0.72)$. Enlargement of the pressure side leg of the horseshoe vortex with the increasing inlet flow angle can be deduced from the increase in the pressure coefficient and pressure gradient. This finding agrees well with the theory of secondary flow presented in the theoretical papers cited above. The maximal pressure gradient in the position $x, y \approx(-45,12)$ was associated with the place where the separation line $\mathfrak{s}_{4}$ begins, see below.

a) $a_{1}$ dep.

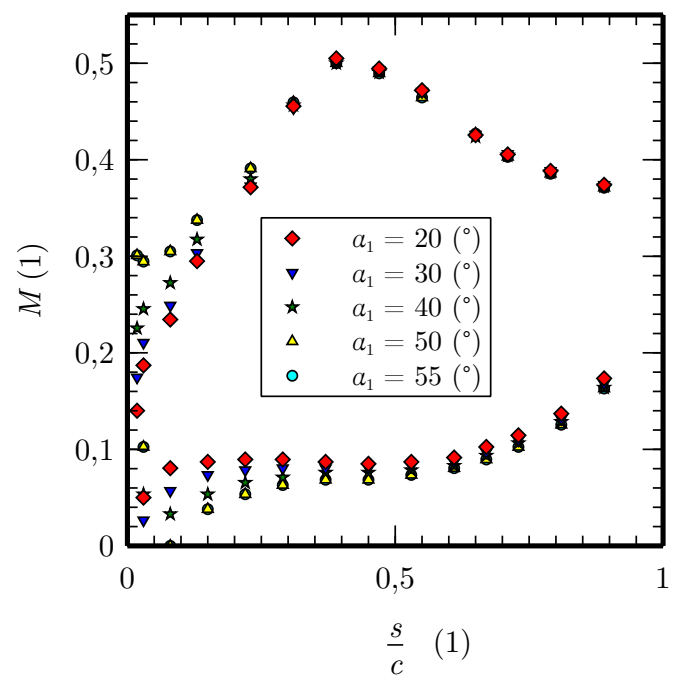

b) $a_{1}$ detail near the blade lading edge

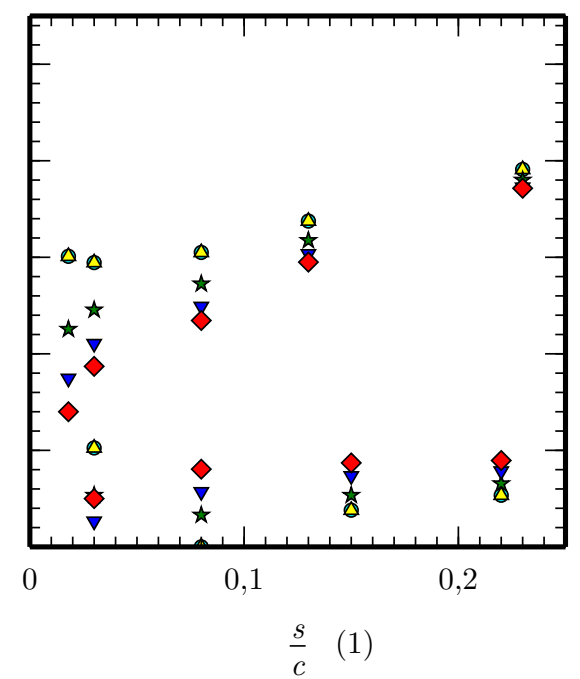

Figure 4: The Mach number distribution at the blade midspan.

The Mach number distributions at the blade mid-span for different inlet flow angles are depicted in Fig. 4. The blade loading around the leading edge rose with increasing inlet flow angle up to $\alpha_{1}=50^{\circ}$. Another increase in the inlet flow angle did not result in another change in blade loading, as is evident.

\subsection{Surface flow visualization}

The surface flow visualizations on the blade suction side are shown in Fig. 5. The inlet flow angle $\alpha_{1}=20^{\circ}$ is depicted in Fig $5 \mathrm{a}$, while the inlet flow angle $\alpha_{1}=55^{\circ}$ is displayed in Fig $5 \mathrm{~b}$. Note, that the increase of the inlet flow angle resulted in the enlargement of the vortex structures which occupied the region in the near-wall. These structures reach up to $10 \%$ and $18 \%$ of the blade span for the nominal regime and overloaded regime, respectively. The evolution of the separation lines $\mathfrak{s}_{2, \mathrm{~s}}$ and $\mathfrak{s}_{4}$ demonstrated the vortex enlargement. The transition to the turbulence in the boundary layer was detected by the visualization as well. This position was localized approximately $20 \mathrm{~mm}$ in front of the blade trailing edge. This conclusion was made not only based on this visualization but thanks to the results obtained in $[13,14]$, where the transition was investigated by infrared thermography as well as numerically on a similar blade. The results presented in those papers ensure the authors about this statement. A slightly different viewing angle caused that it looks like the transition positions in the two figures do not correspond well.

The visualization performed on the end-wall for $\alpha_{1}=55^{\circ}$ is shown in Fig 6 . This visualization is supplemented with a contours line of static pressure coefficient. Unfortunately, the visualization for the nominal case was affected by a blocked tap for the dye injection and therefore this photograph was inapplicable. The contour lines that were connected with the horseshoe vortex pressure leg agree well with the visualization. The horseshoe vortex pressure leg reattached at the point $\mathrm{f}$ the highest pressure coefficient. The separation line $\mathfrak{s}_{4}$ starts here as well. Moreover, this position 
corresponds with the aerodynamic throat. All these factors (or their combination) can be the reason for the large value of the pressure coefficient. Two contour groups positioned approximately in the centre of the blade channel have no physical meaning rather these are artefacts of the pressure tap mesh. The denser mesh should be used in these places to obtain better results.

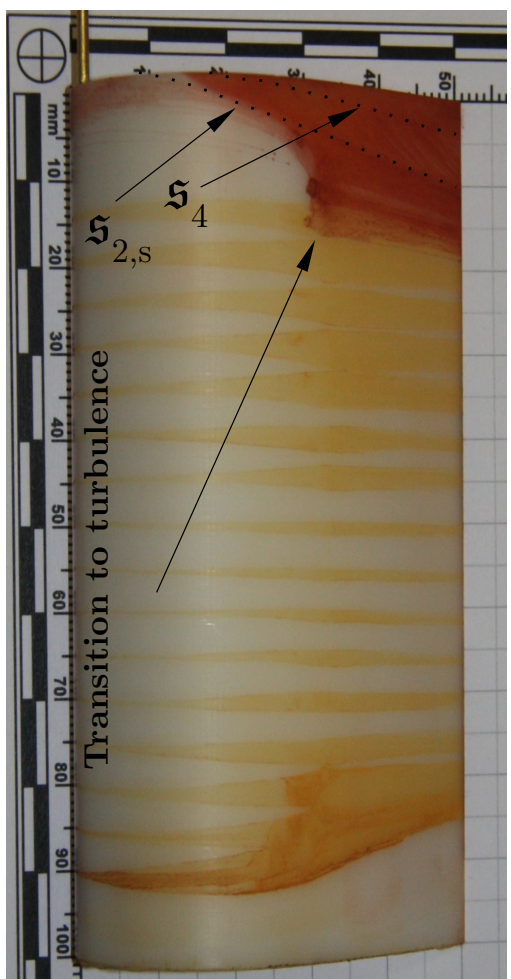

(a) $\alpha_{1}=20^{\circ}$

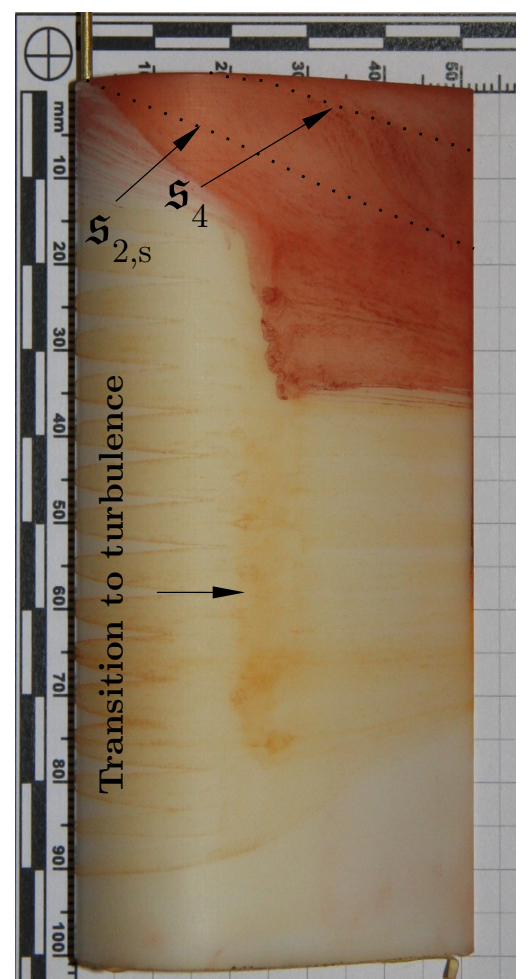

(b) $\alpha_{1}=55^{\circ}$

Figure 5: The surface flow visualization $M_{2, \text { is }}=0.40$ and $R e_{2, \text { is }}=4.5 \times 10^{5}$.

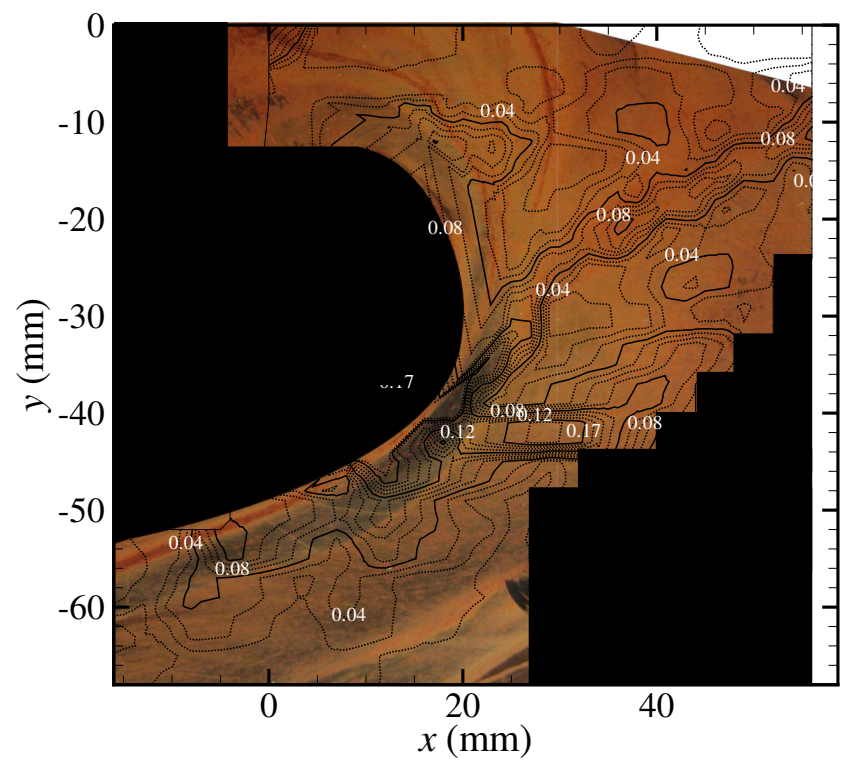

Figure 6: End-wall visualization with the contour of the static pressure coefficient. 


\section{Conclusion}

The experimental research of the near-wall flow in the linear blade cascade was performed. The effect of the inlet flow angle was investigated under constant values of both Reynolds and Mach numbers. The distributions of static pressure gradients, as well as static pressure coefficients, showed that the vortical structures in the blade-to-blade channel grew up with increasing inlet flow angle. The same conclusion can be made based on the visualization experiments. Both results are in agreement with the literature and also coincide with the results that the authors has obtained in the past by the other types of experiments.

This study is part of more complex research focused on secondary flow within a blade row.

\section{Acknowledgment}

These research works were carried out from the funds provided for the "Long-term development of the research organization" (DKRVO) in the form of institutional support of the Ministry of Industry and Trade of the Czech Republic.

\section{References}

[1] Hawthorne W.R.: Rotational Flow Through Cascades, Quart. J. Mech. Appl. Math., vol. 3, (1955) pp. 266-279.

[2] Marris A.W.: The Generation of Secondary Vorticity in an Incompressible Fluid, ASME J. Appl. Mech., vol. 30, (1963) pp. 525-531.

[3] Marris A.W.: On the Generation of Secondary Vorticity Along a Vortex Line, ASME J. Basic Eng., vol. 86, (1964) pp. 815-818.

[4] Marris A.W.: Secondary Flows in an Incompressible Fluid of Varying Density in a Rotating Reference Frame, ASME J. Basic Eng., vol. 88, (1964) pp. 533-537.

[5] Horlock J.H, Lakshminarayana B.: Secondary Flows: Theory, Experiment, and Application in Turbomachinery Aerodynamics, Annu. Rev. Fluid Mech., vol. 5, (1973) pp. 247-280.

[6] Came P.M., Marsh H.: Secondary Flow in Cascades: Two Simple Derivations for the Components of Vorticity, Journal Mechanical Engineering Science, vol. 16, (1974) pp. 391-401.

[7] Hodson H.P., Dominy R.G.: Three-Dimensional Flow in a Low-Pressure Turbine Cascade at Its Design Condition, ASME J. Turbomach., vol. 109, (1987a) pp. 177-185.

[8] Hodson H.P., Dominy R.G.: The Off-Design Performance of a Low-Pressure Turbine Cascade, ASME J. Turbomach., vol. 109, (1987b) pp. 201-209.

[9] Perdichizzi A., Dossena V.: Incidence Angle and Pitch-Chord Effects on Secondary Flows Downstream of a Turbine Cascade, ASME J. Turbomach., vol. 115, (1993) pp. 383-391.

[10] Perdichizzi A.: Mach Number Effect on Secondary Flow Developement Downstream of a Turbine Cascade, ASME J. Turbomach., vol. 112, (1990) pp. 643-651.

[11] Flídr E.: Secondary flow visualization performed for linear blade cascades HR22 a HR22lp, VZLU Technical report R-7104, (2019) - in Czech.

[12] Flídr E. Kladrubský M., Jelínek T.: Visualization study of the secondary flow within a linear blade cascade., TPFM - Conference proceedings, (2019) pp. 77-84.

[13] Flídr E., Jelínek T.: Experimental investigation of the boundary layer transition on the prismatic blade using infrared thermography, XXI. AEaNMiFMaE - Conference proceedings, (2018)

[14] Flídr E., Straka P., Jelínek T.: Experimental and numerical investigation of the boundary layer transition on the prismatic blade, XXIV Biannual Symposium on Measuring Techniques in Turbomachinery Transonic and Supersonic Flow in Cascades and Turbomachines - Conference proceedings, (2018) 